\title{
OPERATOR ALGEBRAS WITH A REDUCTION PROPERTY
}

\author{
JAMES A. GIFFORD
}

(Received 23 February 2004; revised 6 January 2005)

Communicated by G. Willis

\begin{abstract}
Given a representation $\theta: \mathcal{A} \rightarrow \mathcal{B}(H)$ of a Banach algebra $\mathcal{A}$ on a Hilbert space $H, H$ is said to have the reduction property as an $\mathcal{A}$-module if every closed invariant subspace of $H$ is complemented by a closed invariant subspace; $\mathcal{A}$ has the total reduction property if for every representation $\theta: \mathcal{A} \rightarrow \mathcal{B}(H), H$ has the reduction property.

We show that a $C^{*}$-algebra has the total reduction property if and only if all its representations are similar to $*$-representations. The question of whether all $C^{*}$-algebras have this property is the famous 'similarity problem' of Kadison.

We conjecture that non-self-adjoint operator algebras with the total reduction property are always isomorphic to $C^{*}$-algebras, and prove this result for operator algebras consisting of compact operators.
\end{abstract}

2000 Mathematics subject classification: primary 46L05, $46 \mathrm{~L} 07$.

\section{Introduction}

A well-established approach to understanding algebraic objects is to consider their representation theory. The extent to which this approach is successful depends on the characteristics of the algebraic object under consideration, and the nature of the representations chosen.

For instance, finite-dimensional complex representations of finite groups are wellbehaved and lead to a satisfactory classical theory of group representations. One of the reasons that such representations are tractable is that they enjoy the following reduction property: if $\pi: G \rightarrow$ End $V$ is a representation, then every $G$-invariant

The results presented in this paper are extracted from the author's $\mathrm{PhD}$ thesis at the Australian National University.

(C) 2006 Australian Mathematical Society $1446-7887 / 06 \$ A 2.00+0.00$ 
subspace of $V$ is complemented by an invariant subspace. This allows a decomposition of $V$ into a direct sum of irreducible invariant subspaces.

A topological situation where representation theory is indispensible is in $C^{*}$-algebra theory. Here the relevant representations are the $*$-representations, and again these representations enjoy a similar reduction property: if $\mathcal{A}$ is a $C^{*}$-algebra, $\pi: \mathcal{A} \rightarrow$ $\mathcal{B}(H)$ is a $*$-representation and $V \subseteq H$ is $\mathcal{A}$-invariant, then $V^{\perp}$ is also $\mathcal{A}$-invariant. This property is central for the theory of $C^{*}$-algebras.

In this paper we introduce an analogous property for representations of non-selfadjoint operator algebras. Throughout the work all subspaces, submodules and subalgebras will be closed, and all maps will be continuous. By an operator algebra we mean a Banach algebra isomorphic to a subalgebra of $\mathcal{B}(H)$ for some Hilbert space $H$. If $\mathcal{A}$ is an operator algebra and $H$ is a left Banach $\mathcal{A}$-module which is isomorphic to a Hilbert space we say that $H$ is a Hilbertian $\mathcal{A}$-module. When $\mathcal{A} \subseteq \mathcal{B}(H)$ is a subalgebra of $\mathcal{B}(H)$ we take the natural Hilbertian $\mathcal{A}$-module structure on $H$.

DEFINITION 1.1. Let $\mathcal{A}$ be an operator algebra and $H$ a Hilbertian $\mathcal{A}$-module. Then, $H$ is said to have the reduction property if for every closed submodule $V \subseteq H$ there is another closed submodule $W \subseteq H$ with $H=V \oplus W$.

DEFinition 1.2. Let $\mathcal{A} \subseteq \mathcal{B}(H)$ be an operator algebra. If $H$ has the reduction property we say that $\mathcal{A}$ is a reduction algebra.

If $H$ is a Hilbertian $\mathcal{A}$-module the invariant subspace lattice of $H$ will be written Lat $_{H} \mathcal{A}$, or Lat $\mathcal{A}$ when there is no possibility of confusion. The reduction property says that $\mathrm{Lat}_{H} \mathcal{A}$ is what might be called 'topologically complemented'.

The reduction property has been investigated by Rosenoer [12] in the context of operators on a general Banach space, his techniques are similar to the ones used here. However, for our purposes we need something stronger-the reduction property is too weak to imply any self-adjoint structure for an operator algebra. To obtain a rich enough theory we provide two sharpened definitions. When $H$ is an $\mathcal{A}$-module, we denote by $H^{(n)}$ the $n$-fold amplification of $H$ (that is, the left module $H \otimes \mathbb{C}^{n}$ ), and by $H^{(\infty)}$ the countably infinite amplification of $H$.

DefinITION 1.3. Let $\mathcal{A}$ be an operator algebra and $H$ be a Hilbertian $\mathcal{A}$-module. We say that $H$ has the complete reduction property if the amplified module $H^{(\infty)}$ has the reduction property. When $\mathcal{A} \subseteq \mathcal{B}(H)$ and $H$ has the complete reduction property, we say that $\mathcal{A}$ is a complete reduction algebra.

DEFINITION 1.4. Let $\mathcal{A}$ be an operator algebra. We say that $\mathcal{A}$ has the total reduction property if every Hilbertian $\mathcal{A}$-module has the reduction property. For brevity we will also say that $\mathcal{A}$ is a total reduction algebra. 
Note that while both the reduction property and the complete reduction property are tied to a certain representation, the total reduction property is dependent only on the Banach algebra isomorphism class of $\mathcal{A}$. If $\mathcal{A} \subseteq \mathcal{B}(H)$ is an operator algebra, then the total reduction property for $\mathcal{A}$ implies the complete reduction property, which in turn implies the reduction property.

A special form of the reduction property is singled out in the literature-a (weakly closed) operator algebra $\mathcal{A} \subseteq \mathcal{B}(H)$ with the property that $V \in$ Lat $\mathcal{A}$ implies $V^{\perp} \in$ Lat $\mathcal{A}$ is said to be reductive [11]. All von Neumann algebras have this property. The main open question related to reductive algebras is the 'reductive algebra problem': are reductive algebras automatically self-adjoint? A degenerate case of the reduction property occurs when Lat $\mathcal{A}=\{0, H\}$. In this case $\mathcal{A}$ is referred to as a transitive algebra [11]. It is an open question whether a transitive algebra $\mathcal{A} \subseteq \mathcal{B}(H)$ must be all of $\mathcal{B}(H)$.

If $\mathcal{A}$ is an operator algebra and $\theta: \mathcal{A} \rightarrow \mathcal{B}(H)$ is a representation the commutant of the set $\{\theta(a): a \in \mathcal{A}\}$ will be written $\theta(\mathcal{A})^{\prime}$, or $\mathcal{A}^{\prime}$ when there is no danger of confusion. Note that $H$ has the reduction property if and only if every submodule of $H$ is the range of an idempotent operator in $\mathcal{A}^{\prime}$. Since the module maps from $H$ to $H$ are exactly the operators in $\mathcal{A}^{\prime}$, we call such idempotent operators module projections.

The complete reduction property has the desirable feature of offering a uniform bound on the norms of the module projections needed to produce all invariant subspaces.

LEMMA 1.5. Let $\mathcal{A}$ be an operator algebra, and $H$ a Hilbertian $\mathcal{A}$-module with the complete reduction property. There exists $M \geq 1$ so that for any submodule $V \subseteq H$ there is a module projection $p \in \mathcal{A}^{\prime}$ of $H$ onto $V$ with $\|p\| \leq M$.

Proof. For a submodule $V \subseteq H$, let $M(V)$ denote the infimum of the norms of the module projections onto $V$. Suppose that there is a sequence $\left\{V_{i}\right\}$ of submodules with $M\left(V_{i}\right) \rightarrow \infty$. We may consider $V_{i}$ as embedded into the $i$ th component of $H^{(\infty)}$ by $\xi \mapsto \xi \otimes e_{i}$. With this embedding let $V=\sum^{\oplus} V_{i} \subseteq H^{(\infty)}$. Then $V$ is a submodule of $H^{(\infty)}$, and since $H$ has the complete reduction property there is a complementing submodule $U \subseteq H^{(\infty)}$. Let $p: H^{(\infty)} \rightarrow V$ be the corresponding module projection. Let $H_{i}$ denote the copy of $H$ appearing in the $i$ th coordinate position and let $q_{i}: H^{(\infty)} \rightarrow H_{i}$ denote the $i$ th coordinate projection. Then $p_{i}=$ $\left.q_{i} p\right|_{H_{i}}: H_{i} \rightarrow V_{i}$ is a module projection onto $V_{i}$ with $\left\|p_{i}\right\| \leq\|p\|$. However, by assumption $\left\|p_{i}\right\| \geq M\left(V_{i}\right) \rightarrow \infty$. This contradiction establishes the result.

Definition 1.6. Let $\mathcal{A}$ be an operator algebra and $H$ a Hibertian module for $\mathcal{A}$ with the complete reduction property. The smallest $M>0$ such that every submodule of $H$ is the range of a module projection $p$ with $\|p\| \leq M$ is called the projection constant of $H$. 
For total reduction algebras the idea of Lemma 1.5 can be extended by treating more than one representation at once.

LEMMA 1.7. Let $\mathcal{A}$ be an operator algebra with the total reduction property. Then there is an increasing function $K: \mathbb{B}^{+} \rightarrow \mathbb{R}^{+}$such that if $\theta: \mathcal{A} \rightarrow \mathcal{B}(H)$ is a representation of $\mathcal{A}$ and $V \subseteq H$ is a submodule there is a module projection $p: H \rightarrow V$ with $\|p\| \leq K(\|\theta\|)$.

ProOF. Take $C>0$. Suppose that there is a sequence $\left\{\theta_{i}: \mathcal{A} \rightarrow \mathcal{B}\left(H_{i}\right)\right\}$ of representations with $\left\|\theta_{i}\right\| \leq C$ and a sequence $\left\{V_{i} \subseteq H_{i}\right\}$ of submodules such that $K\left(V_{i}\right) \rightarrow \infty$. Consider the direct sum representation $\theta: \mathcal{A} \rightarrow \mathcal{B}\left(\sum^{\oplus} H_{i}\right)$ given by $\theta(a)\left(\xi_{i}\right)=\left(\theta_{i}(a) \xi_{i}\right)$. Then $\|\theta\| \leq C$, and since $\mathcal{A}$ is a total reduction algebra the module $H=\sum^{\oplus} H_{i}$ has the complete reduction property. Thus there is a module projection $p \in \theta(\mathcal{A})^{\prime}$ onto $V=\sum^{\oplus} V_{i}$. As before, if we denote by $q_{i}$ the projection from $H$ onto $H_{i}$, then $p_{i}=\left.q_{i} p\right|_{H_{i}}$ is a projection in $\theta_{i}(\mathcal{A})^{\prime}$ onto $V_{i}$, with $\left\|p_{i}\right\| \leq\|p\|$ for all $i$. This contradiction implies the existence of the function $K$.

\section{A cohomological definition of the total reduction property}

The definition of the total reduction property can be recast into a cohomological setting. The cohomological definition is less illuminating to work with, but has the advantage that it displays the connection between the total reduction property and other notions already in the literature.

For $\theta: \mathcal{A} \rightarrow \mathcal{B}(H)$, a representation of an operator algebra $\mathcal{A}$, the space $\mathcal{B}(H)$ becomes an $\mathcal{A}$-bimodule in the natural way, so we may speak of derivations from $\mathcal{A}$ into $\mathcal{B}(H)$, and of the cohomology group $\mathcal{H}^{1}(\mathcal{A}, \mathcal{B}(H))$ [6].

THEOREM 2.1. An operator algebra $\mathcal{A}$ has the total reduction property if and only if $\mathcal{H}^{1}(\mathcal{A}, \mathcal{B}(H))=0$ for every representation $\theta: \mathcal{A} \rightarrow \mathcal{B}(H)$.

PROOF. Suppose that $\mathcal{A}$ has the total reduction property and that $\theta: \mathcal{A} \rightarrow \mathcal{B}(H)$ is a representation. Let $\delta: \mathcal{A} \rightarrow \mathcal{B}(H)$ be a derivation with respect to $\theta$.

Consider the map $\hat{\theta}: \mathcal{A} \rightarrow \mathcal{B}(H \oplus H)$ given by

$$
\hat{\theta}: a \mapsto\left[\begin{array}{cc}
\theta(a) & \delta(a) \\
0 & \theta(a)
\end{array}\right] .
$$

Matrix multiplication verifies that this is a representation of $\mathcal{A}$, and $H \oplus 0$ is a submodule of $H \oplus H$. Since $\mathcal{A}$ has the total reduction property, there exists a complementary submodule $V$. This subspace must be a closed graph over $0 \oplus H$ and hence is of the form $\{T \eta \oplus \eta: \eta \in 0 \oplus H\}$ for some $T \in \mathcal{B}(H)$. 
Applying the matrix $\hat{\theta}(a)$ to $T \eta \oplus \eta$ gives

$$
\left[\begin{array}{cc}
\theta(a) & \delta(a) \\
0 & \theta(a)
\end{array}\right]\left[\begin{array}{c}
T \eta \\
\eta
\end{array}\right]=\left[\begin{array}{c}
(\theta(a) T+\delta(a)) \eta \\
\theta(a) \eta
\end{array}\right]=\left[\begin{array}{c}
T \theta(a) \eta \\
\theta(a) \eta
\end{array}\right]
$$

by the invariance of $V$. Thus $\delta(a)=T \theta(a)-\theta(a) T$ for all $a \in \mathcal{A}$, showing that $\delta$ is inner and $\mathcal{H}^{1}(\mathcal{A}, \mathcal{B}(H))=0$.

Conversely, suppose $\mathcal{H}^{\prime}(\mathcal{A}, \mathcal{B}(H))=0$ for every representation $\theta: \mathcal{A} \rightarrow \mathcal{B}(H)$. Suppose that $\theta: \mathcal{A} \rightarrow \mathcal{B}(H)$ is a representation of $\mathcal{A}$ and $V \subseteq H$ is a submodule of $H$. The orthogonal decomposition $H=V \oplus V^{\perp}$ gives the matrix form

$$
\theta(a)=\left[\begin{array}{cc}
a_{11} & a_{12} \\
0 & a_{22}
\end{array}\right]
$$

for elements of $\mathcal{A}$. Let us define $\hat{\theta}: \mathcal{A} \rightarrow \mathcal{B}(H)$ and $\delta: \mathcal{A} \rightarrow \mathcal{B}(H)$ by

$$
\hat{\theta}(a)=\left[\begin{array}{cc}
a_{11} & 0 \\
0 & a_{22}
\end{array}\right] \text { and } \delta(a)=\left[\begin{array}{cc}
0 & a_{12} \\
0 & 0
\end{array}\right] .
$$

If $a, b \in \mathcal{A}$ we have

$$
\left[\begin{array}{cc}
a_{11} & a_{12} \\
0 & a_{22}
\end{array}\right]\left[\begin{array}{cc}
b_{11} & b_{12} \\
0 & b_{22}
\end{array}\right]=\left[\begin{array}{cc}
a_{11} b_{11} & a_{11} b_{12}+a_{12} b_{22} \\
0 & a_{22} b_{22}
\end{array}\right]
$$

which shows that $\hat{\theta}$ is a representation of $\mathcal{A}$ and that

$$
\begin{aligned}
\delta(a b) & =\left[\begin{array}{cc}
0 & a_{11} b_{12}+a_{12} b_{22} \\
0 & 0
\end{array}\right] \\
& =\left[\begin{array}{cc}
a_{11} & 0 \\
0 & a_{22}
\end{array}\right]\left[\begin{array}{cc}
0 & b_{12} \\
0 & 0
\end{array}\right]+\left[\begin{array}{cc}
0 & a_{12} \\
0 & 0
\end{array}\right]\left[\begin{array}{cc}
b_{11} & 0 \\
0 & b_{22}
\end{array}\right] \\
& =\hat{\theta}(a) \delta(b)+\delta(a) \hat{\theta}(b) .
\end{aligned}
$$

Thus $\delta$ is a derivation with respect to the representation $\hat{\theta}$. Since $\mathcal{H}^{\prime}(\mathcal{A}, \mathcal{B}(H))=0$ where $\mathcal{B}(H)$ is now considered as a bimodule via $\hat{\theta}$, we have $\delta(a)=\hat{\theta}(a) T-T \hat{\theta}(a)$ for some $T \in \mathcal{B}(H)$. Writing

$$
T=\left[\begin{array}{ll}
T_{11} & T_{12} \\
T_{21} & T_{22}
\end{array}\right]
$$

and expanding the identity $\delta(a)=\hat{\theta}(a) T-T \hat{\theta}(a)$ in components gives $a_{12}=a_{11} T_{12}-$ $T_{12} a_{22}$. Then the subspace

$$
\left\{\left[\begin{array}{c}
-T_{12} \eta \\
\eta
\end{array}\right]: \eta \in V^{\perp}\right\}
$$

is a $\theta(\mathcal{A})$-invariant complement to $V$ and hence $\mathcal{A}$ is a total reduction algebra. 
This characterisation of total reduction algebras should be compared to the definition of amenability of Banach algebras. Recall that a Banach algebra $\mathcal{A}$ is amenable if $\mathcal{H}^{1}\left(\mathcal{A}, X^{*}\right)=0$ for all dual Banach $\mathcal{A}$-bimodules $X^{*}$. The bimodules $\mathcal{B}(H)$ which arise above are in fact dual bimodules, a fact which can be verified by considering the space $T C(H)$ of trace-class operators on $H$. It is well-known that $T C(H)$ is the predual to $\mathcal{B}(H)$ and we have the canonical inclusion $T C(H) \subseteq \mathcal{B}(H)^{*}$. It is easily seen that $T C(H)$ is a submodule of the dual module $\mathcal{B}(H)^{*}$ and that the module action on $\mathcal{B}(H)$ is itself dual to this module action on $T C(H)$. This gives the following proposition.

PROPOSITION 2.2. Let $\mathcal{A}$ be an amenable operator algebra. Then $\mathcal{A}$ has the total reduction property.

\section{3. $C^{*}$-algebras and the reduction property}

We have observed that if $\mathcal{A}$ is a $C^{*}$-algebra and $\pi: \mathcal{A} \rightarrow \mathcal{B}(H)$ is a $*$-representation then $H$ has the reduction property as an $\mathcal{A}$-module. For non-* representations the situation is not so simple.

Let $\mathcal{A}$ be an operator algebra. Two representations $\theta: \mathcal{A} \rightarrow \mathcal{B}(H)$ and $\psi$ : $\mathcal{A} \rightarrow \mathcal{B}(H)$ are said to be similar if there is an isomorphism $S: H \rightarrow H$ with $\theta(a)=\psi^{S}(a)=S^{-1} \psi(a) S$ for all $a \in \mathcal{A}$. In the case where $\mathcal{A}$ is a $C^{*}$-algebra, we say that $\mathcal{A}$ has the similarity property if every representation of $\mathcal{A}$ is similar to a *-representation. An intriguing open question in the theory of $C^{*}$-algebras is the similarity question $[7,10]$ :

QUESTION 3.1 (Similarity Question). Does every $C^{*}$-algebra have the similarity property?

The following is almost immediate.

LEMMA 3.2. Let $\mathcal{A}$ be a $C^{*}$-algebra with the similarity property. Then $\mathcal{A}$ has the total reduction property.

There are several partial results to the similarity question, most of which can be found in Pisier's book [10]. Many of these find restrictions on either the algebra or the module which force the representation to be similar to a $*$-representation. The most prominent result of the latter type is Haagerup's result for finitely generated modules $[5,10]$.

THEOREM 3.3. Let $\mathcal{A}$ be a $C^{*}$-algebra and $\theta: \mathcal{A} \rightarrow \mathcal{B}(H)$ a representation of $\mathcal{A}$. If there is a finite set $\left\{\xi_{i}\right\}$ of vectors in $H$ such that the submodule generated by $\left\{\xi_{i}\right\}$ is $H$, then $\theta$ is similar to a $*$-representation. 
In general, it is not known whether the size of the similarity obtained in this result can be bounded in terms of $\|\theta\|$ alone. Indeed, a simple ultrafilter argument shows that establishing such a bound is equivalent to solving the similarity problem [10]. However, when $\mathcal{A}$ has the total reduction property the norms of the similarities can be bounded in terms of $\|\theta\|$ using the projection constant function obtained from Lemma 1.7. The proof of this fact is adapted from the proof of the complemented subspaces theorem which appears in [3].

LEMMA 3.4. Let $\mathcal{A}$ be a $C^{*}$-algebra with the total reduction property, and let $\theta: \mathcal{A} \rightarrow \mathcal{B}(H)$ be a representation which is similar to a $*$-representation. If $K$ is the projection constant function of Lemma 1.7 then there is a similarity $S$ so that $\theta^{S}$ is a *-representation and $\|S\|\left\|S^{-1}\right\| \leq 128 K(\|\theta\|)^{2}$.

Proof. Let us say that a Hilbertian $\mathcal{A}$-module $H^{\prime}$ is a $*$-module for $\mathcal{A}$ if the corresponding representation is a $*$-representation. Let

$$
\alpha=\inf \left\{\|S\|\left\|S^{-1}\right\|: S \text { is a module isomorphism from } H \text { onto a } * \text {-module }\right\} .
$$

By assumption $\alpha<\infty$ and we may find a $*$-module $H^{\prime}$ and contractive module isomorphism $S: H \rightarrow H^{\prime}$ with $\left\|S^{-1}\right\| \leq 2 \alpha$. Since $H^{\prime}$ is a $*$-module the corresponding representation $\mathcal{A} \rightarrow \mathcal{B}\left(H^{\prime}\right)$ is contractive and the representation $\theta^{\prime}: \mathcal{A} \rightarrow \mathcal{B}(H) \oplus \mathcal{B}\left(H^{\prime}\right)$ has $\left\|\theta^{\prime}\right\| \leq\|\theta\|$ (assuming $\theta \neq 0$ ). Thus $H \oplus H^{\prime}$ has the reduction property with projection constant $M \leq K(\|\theta\|)$ and consequently for any $\mu \in \mathbb{R}^{+}$there is a module projection $p$ from $H \oplus H^{\prime}$ onto $\mathrm{Gr} \mu S=\{\xi \oplus \mu S \xi: \xi \in H\}$ with $\|p\| \leq M$. Writing $p$ in matrix components reveals that $p$ has the form

$$
p=\left[\begin{array}{cc}
1+R \mu S & -R \\
\mu S(1+R \mu S) & -\mu S R
\end{array}\right]
$$

for some module map $R: H^{\prime} \rightarrow H$. Since $\|p\| \leq M$, it follows that $\|R\| \leq M$ and $\|\mu S(1+R \mu S)\| \leq M$.

Now consider the module map $T: H \rightarrow H^{\prime} \oplus H^{\prime}$ given by

$$
T \xi=\frac{1}{2} S \xi \oplus \frac{1}{2 M}(\mu S(1+R \mu S) \xi) .
$$

This is a contractive module isomorphism onto some closed submodule of $H^{\prime} \oplus H^{\prime}$. Since $H^{\prime}$ is a $*$-module for $\mathcal{A}, H^{\prime} \oplus H^{\prime}$ is also a $*$-module and hence any submodule of $H^{\prime} \oplus H^{\prime}$ is a $*$-module. By the definition of $\alpha$, this means that there is $\xi_{0} \in H$ with $\left\|\xi_{0}\right\|=1$ and $\left\|T \xi_{0}\right\| \leq 2 \alpha^{-1}$.

Suppose that $\left\|S \xi_{0}\right\| \leq(2 M \mu)^{-1}$. From the second term in the definition of $T$ we see that $\left\|T \xi_{0}\right\| \geq \mu / 8 M \alpha$. Since we know that $2 \alpha^{-1} \geq\left\|T \xi_{0}\right\|$ this is impossible if we choose $\mu>16 M$. 
Thus if $\mu=16 M+\epsilon$ we must have $\left\|S \xi_{0}\right\|>(2 M \mu)^{-1}=\left(32 M^{2}+2 M \epsilon\right)^{-1}$. Then the first term in the definition of $T$ gives the inequality

$$
2 \alpha^{-1} \geq 2^{-1}\left\|S \xi_{0}\right\|>\left(64 M^{2}+4 M \epsilon\right)^{-1},
$$

and hence $\alpha \leq 128 M^{2}$.

This result allows us to pass from finitely generated modules to general modules, as follows.

PROPOSITION 3.5. Let $\mathcal{A}$ be a $C^{*}$-algebra with the total reduction property. Then every representation of $\mathcal{A}$ is similar to a $*$-representation.

ProOF. Let $\theta: \mathcal{A} \rightarrow \mathcal{B}(H)$ be a representation of $\mathcal{A}$. From Haagerup's result we know that every finitely generated subrepresentation of $\theta$ is similar to a *-representation. To pass to the full representation consider the set $\Lambda$ of finite subsets of $H$, and let $\mathcal{F}$ denote an ultrafilter on $\Lambda$ which contains the filterbase of sets of the form $\left\{\lambda: \lambda \supseteq \lambda_{0}\right\}$. For each $\lambda=\left\{\xi_{i}\right\} \in \Lambda$, let $H_{\lambda}$ be the submodule of $H$ generated by $\left\{\xi_{i}\right\}$. Then by Lemma 3.4 there are contractive similarities $S_{\lambda} \in \mathcal{B}\left(H_{\lambda}\right)$ with $\left\|S_{\lambda}{ }^{-1}\right\| \leq 128[K(\|\theta\|)]^{2}$ such that applying $S_{\lambda}$ makes $\left.\theta\right|_{H_{\lambda}}$ a $*$-representation. In particular, if $\xi, \eta \in \lambda \in \Lambda$, then $\left(S_{\lambda} \theta(a) \xi \mid S_{\lambda} \eta\right)=\left(S_{\lambda} \xi \mid S_{\lambda} \theta\left(a^{*}\right) \eta\right)$ for all $a \in \mathcal{A}$. We define a new inner product on $H$ by

$$
(\xi \mid \eta)_{\text {new }}=\lim _{\mathcal{F}}\left(S_{\lambda} \xi \mid S_{\lambda} \eta\right)
$$

Routine verification shows that this defines an inner product norm on $H$ equivalent to the original norm, and $\theta$ becomes a $*$-representation with respect to this inner product.

COROLLARY 3.6. The total reduction property is equivalent to the similarity property for $C^{*}$-algebras.

Combining Proposition 2.2 and Corollary 3.6 gives a new proof of the known fact that amenable $C^{*}$-algebras have the similarity property.

Corollary 7.14 of [10] shows for any Hilbert space $H$, that $\mathcal{B}(H)$ has the similarity property. Thus $\mathcal{B}(H)$ always has the total reduction property, and for infinite dimensional $H$ this provides an example of a total reduction algebra which does not have the approximation property and is not amenable [8].

\section{Structure of reduction algebras}

We propose the following two conjectures concerning the structure of operator algebras with either the complete reduction property or the total reduction property. 
CONJECTURE 4.1. Every total reduction algebra is isomorphic to a $C^{*}$-algebra.

CONJECTURE 4.2. Every weakly closed complete reduction algebra is similar to a $C^{*}$-algebra.

The second conjecture can be considered as a non-self-adjoint analogue of the reductive algebra problem. Note that if $\mathcal{A} \subseteq \mathcal{B}(H)$ is a total reduction algebra and is isomorphic to a $C^{*}$-algebra, Corollary 3.6 shows that $\mathcal{A}$ is similar to a $C^{*}$-algebra. It is not difficult to construct examples to show that the hypothesis of weak closure is necessary in Conjecture 4.2.

Any solution to Conjecture 4.2 must be equivalent to the following procedure.

(i) Use the fact that Lat ${ }_{H} \mathcal{A}$ is topologically complemented to find a similarity $S \in \mathcal{B}(H)$ so that Lat $\mathcal{A}^{S}$ is orthogonally complemented. That is, renorm $H$ with an equivalent Hilbert space norm so that under this norm Lat ${ }_{H} \mathcal{A}$ becomes orthogonally complemented.

(ii) Having reduced to the case where Lat $\mathcal{A}^{S}$ is orthogonally complemented, show that $\mathcal{A}^{S}$ is self-adjoint.

For, if $\mathcal{A}$ is weakly closed and similar to a $C^{*}$-algebra, then certainly the similarity $S$ in step (i) can be found. Moreover, if $S$ is such that Lat $\mathcal{A}^{S}$ is orthogonally complemented, then since $\mathcal{A}=\operatorname{Alg}$ Lat $\mathcal{A}$ we see that $\mathcal{A}^{S}$ is self-adjoint, and so step (ii) can be established.

The first step can be considered as a module analogue of the complemented subspaces theorem from Banach space theory [3]. This theorem states: if every subspace of a Banach space $X$ is complemented then $X$ is isomorphic to a Hilbert space. If we write Lat $X$ for the lattice of subspaces of $X$, the theorem becomes: if Lat $X$ is topologically complemented, then $X$ can be renormed by an equivalent Hilbert space norm so that Lat $X$ becomes orthogonally complemented. The connection with step (i) above is substantiated in Lemma 5.8 below, where we emulate the Banach space proof in a module context.

Once step (i) has been achieved $\mathcal{A}^{S}$ is a reductive algebra. The reductive algebra problem demonstrates that step (ii) will not in general be a trivial step. In particular, if Lat $\mathcal{A}=\{0, H\}$ then step (i) is automatically obtained and we are dealing with a transitive algebra. To make progress we need to restrict attention to cases where the reductive and transitive algebra problems cannot cause difficulties.

A theorem of Lomonosov states that if $B \subseteq \mathcal{B}(H)$ is a (not-necessarily closed) algebra of operators with Lat $B=\{0, H\}$ and $B$ contains a non-zero compact operator, then $B$ contains a compact operator with positive spectral radius. A corollary of this is that if $\mathcal{A} \subseteq \mathcal{K}(H)$ is an operator algebra of compact operators with Lat $\mathcal{A}=\{0, H\}$, then $\mathcal{A}=\mathcal{K}(H)$ [11]. This theorem effectively removes the transitive algebra problem for algebras with non-zero compact operators and allows us to completely understand 
the structure of complete reduction algebras consisting solely of compact operators.

The remainder of the paper is devoted to establishing the following result.

THEOREM 4.3. Let $\mathcal{A} \subseteq \mathcal{K}(H)$ be a complete reduction algebra. Then $\mathcal{A}$ is similar to a $C^{*}$-algebra.

$C^{*}$-algebras of compact operators are described by a Wedderburn-like structure theorem [1]. If $\mathcal{A} \subseteq \mathcal{K}(H)$ is a $C^{*}$-algebra of compact operators, then there is a family of Hilbert spaces $\left\{V_{\lambda}\right\}$ indexed by some set $\Lambda$ and an integer-valued function $\lambda \mapsto n_{\lambda}$, so that $H$ is isometrically isomorphic to $\sum^{\oplus} V_{\lambda} \otimes \mathbb{C}^{n_{\lambda}}$ and $\mathcal{A}$ is isometrically isomorphic to the $C^{*}$-algebra $\sum^{c_{0}} \mathcal{K}\left(V_{\lambda}\right) \otimes 1_{n_{\lambda}}$.

Considering a non-self-adjoint complete reduction algebra $\mathcal{A} \subseteq \mathcal{K}(H)$, the next few results lead up to Theorem 4.10 which will allow us to reduce the problem to the case where $\mathcal{A}^{\prime \prime}$ contains no proper central projections. From the above description of $C^{*}$-algebras of compact operators, this should correspond to the case where $\mathcal{A}$ is similar to $\mathcal{K}(V) \otimes 1_{n}$ for some Hilbert space $V$ and integer $n$.

If $\mathcal{A} \subseteq \mathcal{K}(H)$ is an operator algebra with the reduction property, the submodule $\overline{A H}$ is complemented by some $V \in$ Lat $\mathcal{A}$. Then $\mathcal{A} V \subseteq \overline{\mathcal{A H}} \cap V=\{0\}$, so $\mathcal{A}$ annihilates $V$. Applying a similarity, we may assume that $\overline{\mathcal{A} H} \perp V$. In terms of showing that $\mathcal{A}$ is similar to a $C^{*}$-algebra, it is clearly equivalent to show that the isometrically isomorphic restricted algebra $\left.\mathcal{A}\right|_{\overline{A H}}$ is similar to a $C^{*}$-algebra. Thus we may reduce without loss of generality to the case where $\overline{\mathcal{A} H}=H$. In this case we say that $\mathcal{A}$ acts nondegenerately on $H$. For the remainder of this paper we assume that all operator algebras act nondenegerately.

LEMMA 4.4. Let $\mathcal{A} \subseteq \mathcal{B}(H)$ be an operator algebra with the complete reduction property, and let $P$ be the set of central projections of $\mathcal{A}^{\prime \prime}$. Then $P$ is bounded. Moreover, there exists a similarity $S$ of $H$ which makes all the central projections self-adjoint.

ProOF. Let $M$ be the projection constant of $H$ and suppose $p \in P$. Then $p H$ is a submodule of $H$. By Lemma 1.5 there is a projection $q \in \mathcal{A}^{\prime}$ with $\|q\| \leq M$ and $q H=p H$. Now $q \in \mathcal{A}^{\prime}, p \in \mathcal{A}^{\prime \prime}$ and they share the same range space, so $q=p q=q p=p$, giving $\|p\| \leq M$.

To show that a similarity can be found which orthogonalises these central projections, consider the abelian group obtained from the involutive operators $G=$ $\{1-2 p: p \in P\}$. This is a bounded abelian group, and so there is a similarity $S$ under which $G$ becomes a unitary group [9]. However, if $(1-2 p)^{S}$ is unitary then $p^{s}$ is self-adjoint. 
LEMma 4.5. Let $\mathcal{A}$ be an operator algebra. Suppose that $H$ is a Hilbertian $\mathcal{A}$ module with the reduction property and that $\overline{\mathcal{A} H}=H$. Then $\xi \in \overline{\mathcal{A}} \xi$ for all $\xi \in H$.

Proof. For every $\xi \in H$ the space $\overline{\mathcal{A} \xi}$ is $\mathcal{A}$-invariant. The reduction property yields $V \in$ Lat $\mathcal{A}$ with $H=\overline{\mathcal{A} \xi} \oplus V$ as $\mathcal{A}$-modules, giving a decomposition $\xi=\xi_{1} \oplus \xi_{2}$ into the respective components. We have $\mathcal{A} \xi=\mathcal{A}\left(\xi_{1} \oplus \xi_{2}\right) \subseteq \overline{\mathcal{A}} \xi$, so $\mathcal{A} \xi_{2}=0$. We wish to show that $\xi_{2}=0$. Consider the subspace $W=\{\eta: \mathcal{A} \eta=0\}$. This is a closed $\mathcal{A}$-module containing $\xi_{2}$ and using the reduction property there is $U \in$ Lat $\mathcal{A}$ with $H=W \oplus U$. Then by the nondegeneracy assumption $H=\overline{\mathcal{A} H}=\overline{\mathcal{A} W \oplus \mathcal{A} U} \subseteq$ $\overrightarrow{\mathcal{A} U}$, implying that $U=H$. Thus $W=\{0\}$ and so $\xi_{2}=0$.

COROLLARY 4.6. Let $\mathcal{A} \subseteq \mathcal{B}(H)$ be an operator algebra such that $H$ has the complete reduction property and $\overline{\mathcal{A H}}=H$. For any $1 \leq n \leq \infty$ and any $\xi \in H^{(n)}$ we have $\xi \in \overline{A^{(n)} \xi}$.

Proof. It follows from $\overline{\mathcal{A H}}=H$ that $\overline{A^{(n)} H^{(n)}}=H^{(n)}$. Then Lemma 4.5 gives the result.

THEOREM 4.7. Let $\mathcal{A}$ be an operator algebra, and suppose $\theta: \mathcal{A} \rightarrow \mathcal{B}(H)$ is a representation of $\mathcal{A}$ such that $H$ has the complete reduction property. If $\overline{\mathcal{A} H}=H$ then $\overline{\theta(\mathcal{A})}^{\sigma}=\mathcal{A}^{\prime \prime}$. Here ${\overline{\theta(\mathcal{A})^{\sigma}}}^{w}$ refers to the $\sigma$-weak closure of $\theta(\mathcal{A})$.

Proof. It is immediate that $\overline{\theta(\mathcal{A})}^{\sigma w} \subseteq \mathcal{A}^{\prime \prime}$. For the converse, take $T \in \mathcal{A}^{\prime \prime}$ and a sequence $\xi=\left(\xi_{i}\right) \in H^{(\infty)}$. We will show that for any $\epsilon>0$ there is $a \in \mathcal{A}$ with $\left\|T^{(\infty)} \xi-a^{(\infty)} \xi\right\|<\epsilon$. This will demonstrate that the $\sigma$-strong closure of $\theta(\mathcal{A})$ contains $\mathcal{A}^{\prime \prime}$; since the $\sigma$-weak topology is weaker than the $\sigma$-strong topology, the result will follow.

Using Corollary 4.6 the vector $\xi$ lies in $\overline{\mathcal{A}^{(\infty)} \xi}$. The reduction property gives a projection in $\left(\mathcal{A}^{(\infty)}\right)^{\prime}$ onto $\overline{\mathcal{A}^{(\infty)} \xi}$. Since $T \in \mathcal{A}^{\prime \prime}$, it follows that $T^{(\infty)} \in\left(\mathcal{A}^{(\infty)}\right)^{\prime \prime}$, and so $T^{(\infty)} \xi \in \overline{\mathcal{A}^{(\infty)} \xi}$. Consequently, there is $a \in \mathcal{A}$ with $\left\|T^{(\infty)} \xi-a^{(\infty)} \xi\right\|<\epsilon$.

COROLlARY 4.8. Let $\mathcal{A} \subseteq \mathcal{B}(H)$ be a nondegenerate operator algebra with the complete reduction property. Then $\mathcal{A}^{\prime \prime}=\overline{\mathcal{A}}^{\sigma w}$.

For $C^{*}$-algebras this is the famous von Neumann double commutant theorem [1]. Our proof is a modification of the usual $C^{*}$-algebra proof.

LEMMA 4.9. Suppose that $B \subseteq \mathcal{K}(H)$ is an algebra of compact operators acting nondegenerately on $H$. If $R$ is an abelian von Neumann algebra commuting with $B$, then $R$ is generated as a weakly closed algebra by its minimal projections. 
PROOF. Since $R$ is self-adjoint, it commutes not only with $B$ but also with $\left\{b^{*}\right.$ : $b \in B\}$ and hence with the $C^{*}$-algebra generated by $B$, which we write $C^{*}(B)$. This is a $C^{*}$-algebra consisting of compact operators acting nondegenerately on $H$, so by the structure theory for $C^{*}$-algebras of compact operators there is a family $H_{\gamma}$ of Hilbert spaces and multiplicity function $\gamma \mapsto n_{\gamma}$ such that $C^{*}(B)$ is unitarily isomorphic to $\sum_{\gamma}^{c_{0}} \mathcal{K}\left(H_{\gamma}\right) \otimes 1_{n_{\gamma}}$. The commutant $M=C^{*}(B)$ may be read off from this as $M=\sum_{\gamma}^{\ell^{\infty}} 1_{H_{\gamma}} \otimes M_{n_{\gamma}} \cong \sum_{\gamma}^{\ell^{\infty}} M_{n_{\gamma}}$. We write $M_{\gamma}$ for the $\gamma$ th summand. The projections from $M$ onto $M_{\gamma}$ are central projections of $M$. Let $D$ be a maximal abelian self-adjoint subalgebra of $M$ containing $R$. The maximality of $D$ implies that $D$ contains the centre of $M$, and so $D=\sum_{\gamma}^{\ell^{\infty}}\left(D \cap M_{\gamma}\right)$, where $D \cap M_{\gamma}$ is a maximal abelian self-adjoint subalgebra of $M_{\gamma}$. The maximal abelian self-adjoint subalgebras of $M_{\gamma}$ are isomorphic to $\ell^{\infty}\left(n_{\gamma}\right)$, and so $D \cong \sum^{\ell^{\infty}} \ell^{\infty}\left(n_{\gamma}\right) \cong \ell^{\infty}(\Omega)$ for some index set $\Omega$. This allows us to think of $R$ as a self-adjoint subalgebra of $\ell^{\infty}(\Omega)$. Let $\Lambda$ be the set of equivalence classes of $\Omega$ under the relation

$$
\omega_{1} \equiv \omega_{2} \Longleftrightarrow r\left(\omega_{1}\right)=r\left(\omega_{2}\right) \text { for all } r \in R .
$$

By this construction we may think of $R$ as a self-adjoint subalgebra of $\ell^{\infty}(\Lambda)$. For $\lambda \in \Lambda$ consider the set

$$
P_{\lambda}=\{p \in R: p \text { is idempotent, } p(\lambda)=1\} .
$$

Since the projections in $R$ form a complete Boolean algebra, $P_{\lambda}$ will possess an infimum, $p_{\lambda}$ say, which must be given by a characteristic function of a subset of $\Lambda$ containing $\lambda$. Because $R$ is generated by its projections and separates the points of $\Lambda$, we have $p_{\lambda}=1_{\{\lambda\}}$, and so $R=\ell^{\infty}(\Lambda)$. The lattice of projections of $R$ is thus $\mathcal{P}(\Lambda)$, which is atomic with atoms $l_{\{\lambda\}}$. These are exactly the minimal projections of $R$.

THEOREM 4.10. Suppose $\mathcal{A} \subseteq \mathcal{K}(H)$ is a nondegenerate complete reduction algebra. Then the central idempotents of $\mathcal{A}^{\prime \prime}$ form a complete atomic Boolean algebra. The atoms are exactly the minimal central projections of $\mathcal{A}^{\prime \prime}$. Writing $\left\{p_{\dot{\lambda}}\right\}$ for this set of minimal idempotents, the algebras $\mathcal{A}_{\lambda}=p_{\lambda} \mathcal{A}$ are closed two-sided ideals of $\mathcal{A}$ and considering $\mathcal{A}_{\lambda}$ as an operator algebra on $p_{\lambda} H$, the bicommutant $\mathcal{A}_{\lambda}^{\prime \prime}$ has no proper central projections. Finally, $\mathcal{A} \cong \sum^{c_{0}} \mathcal{A}_{\lambda}$.

Proof. Using Lemma 4.4 we may assume that the central projections of $\mathcal{A}^{\prime \prime}$ are self-adjoint. Let $R$ be the abelian von Neumann algebra generated by these central projections. Lemma 4.9 shows that $R$ is generated by its minimal projections. With $\left\{p_{\lambda}\right\}$ and $H_{\lambda}$ as specified, the nondegeneracy assumption implies $H=\sum^{\oplus} H_{\lambda}$ and $\sum p_{\lambda}=1$ (strong convergence).

Let us write $\mathcal{A}_{\lambda}$ for the (possibly non-closed) algebra $p_{\lambda} \mathcal{A} \subseteq \mathcal{B}\left(H_{\lambda}\right)$. Suppose that $p \in \mathcal{A}_{\lambda}^{\prime \prime} \subseteq \mathcal{B}\left(H_{\lambda}\right)$ is a central projection of $\mathcal{A}_{\lambda}^{\prime \prime}$ for some $\lambda \in \Lambda$. Then $p p_{\lambda}$ is central 
for $\mathcal{A}^{\prime \prime}$, and $0 \leq p p_{\lambda} \leq p_{\lambda}$. Since $p_{\lambda}$ is an atom in the lattice of central projections of $\mathcal{A}$, either $p=0$ or $p=p_{\lambda}$. In either case, $p$ is not a proper central projection of $\mathcal{A}_{\lambda}^{\prime \prime}$.

We claim that $\mathcal{A}_{\lambda}$ is a closed ideal of $\mathcal{A}$. To see that $p_{\lambda} \mathcal{A} \subseteq \mathcal{A}$, recall the duality $\mathcal{K}(H)^{* *}=(T C(H))^{*}=\mathcal{B}(H)$, where $T C(H)$ is the space of trace class operators on $H$. With this duality, the $\sigma$-weak topology on $\mathcal{B}(H)$ coincides with the $\sigma(\mathcal{B}(H), T C(H))$-topology. Suppose then that $p_{\lambda} a \notin \mathcal{A}$ for some $a \in \mathcal{A}$. Since $p_{\lambda} a$ is compact, by the Hahn-Banach theorem there is $f \in T C(H)$ with $\langle f, \mathcal{A}\rangle=0$ and $\left\langle f, p_{\lambda} a\right\rangle=1$. However, since by Theorem 4.7, $p_{\lambda} a \in \mathcal{A}^{\prime \prime}=\overline{\mathcal{A}}^{\sigma w}$, there is a net $\left\{b_{\mu}\right\}$ in $\mathcal{A}$ with $b_{\mu} \stackrel{\sigma a \prime}{\longrightarrow} p_{\lambda} a$. Since $f \in T C(H), f$ is $\sigma(\mathcal{B}(H), T C(H))$-continuous (that is, $\sigma$-weak-continuous) and we have $0=\left\langle f, b_{\mu}\right\rangle \rightarrow\left\langle f, p_{\lambda} a\right\rangle=1$. This contradiction implies that $p_{\lambda} \mathcal{A} \subseteq \mathcal{A}$ and that $p_{\lambda} \mathcal{A}$ is norm closed (being the range of the projection $\left.\left.p_{\lambda}\right|_{\mathcal{A}}\right)$. Since $p_{\lambda}$ is central, $p_{\lambda} \mathcal{A}$ is a two-sided ideal of $\mathcal{A}$.

Let us write $\sum^{c^{\infty} 0} \mathcal{A}_{\lambda}$ for the algebraic direct sum (that is, taking elements with only finitely many non-zero terms). The norm closure of $\sum^{c_{\infty}} \mathcal{A}_{\lambda}$ is $\sum^{c_{0}} \mathcal{A}_{\lambda}$. Since the idempotents $\left\{p_{\lambda}\right\}$ are self-adjoint and mutually orthogonal, we have the isometric embedding $\sum^{c_{\infty}} \mathcal{A}_{\lambda} \subseteq \mathcal{A}$ and as $\mathcal{A}$ is norm closed this implies that $\sum^{c_{0}} \mathcal{A}_{\lambda} \subseteq \mathcal{A}$. On the other hand, as $\sum p_{\lambda}=1$ the equality $a=\sum p_{\lambda} a$ holds for all $a \in \mathcal{A}$. The compactness of $a$ then implies that $\lambda \mapsto\left\|p_{\lambda} a\right\| \in c_{0}(\Lambda)$ and so $\mathcal{A} \subseteq \sum^{c_{0}} \mathcal{A}_{\lambda}$.

This result will allow us to reduce attention to the case where $\mathcal{A}^{\prime \prime}$ contains no proper central projections.

The next few lemmas apply to general complete reduction algebras rather than only those consisting of compact operators. Let $\mathcal{A} \subseteq \mathcal{B}(H)$ denote a complete reduction algebra acting on Hilbert space $H$. If $V$ and $W$ are two submodules of $H$, we consider the space of module maps from $V$ to $W$. We define a relation $\leadsto$ on the set of submodules of $H$ as follows: $V \leadsto W$ if and only if there is a non-zero module map from $V$ to $W$.

LEMMA 4.11. Let $\mathcal{A} \subseteq \mathcal{B}(H)$ be a complete reduction algebra and suppose $V_{1}, V_{2}$ are submodules of $H$ with $V_{2} \leadsto V_{1}$. Then $V_{1} \leadsto V_{2}$.

ProOF. We may assume that $V_{1} \cap V_{2}=\{0\}$ by replacing $H$ with $H \otimes \mathbb{C}^{2}$ if necessary and replacing $V_{1}$ with $V_{1} \otimes e_{1}$ and $V_{2}$ with $V_{2} \otimes e_{2}$. This device also ensures that $V_{1}+V_{2}$ is closed.

Suppose that $V_{1} \not 4 V_{2}$. Consider the subrepresentation $\theta$ of $\mathcal{A}$ on $V_{1} \oplus V_{2}$. The elements of $\theta(\mathcal{A})^{\prime}$ have the matrix form

$$
\left[\begin{array}{cc}
b_{11} & 0 \\
b_{21} & b_{22}
\end{array}\right]
$$

where each $b_{i j}: V_{j} \rightarrow V_{i}$ is a module map. Since $V_{2} \leadsto V_{1}$ there is a non-zero module 
map $T: V_{2} \rightarrow V_{1}$. For $\lambda \in \mathbb{R}^{+}$, consider the matrix

$$
\left[\begin{array}{cc}
1 & 0 \\
\lambda T & 0
\end{array}\right] \in \theta(\mathcal{A})^{\prime}
$$

This is a projection onto $\operatorname{Gr} \lambda T$ with kernel $V_{2}$, and it is the only projection onto $\operatorname{Gr} \lambda T$ of the form given for elements of $\theta(\mathcal{A})^{\prime}$. However, as $\lambda \rightarrow \infty$ the norm of this projection goes to $\infty$. Then Lemma 1.5 gives a contradiction.

LEMMA 4.12. Let $\mathcal{A} \subseteq \mathcal{B}(H)$ be a complete reduction algebra and denote by $B$ the closed algebra generated by $\mathcal{A}$ and $\mathcal{A}^{\prime}$. Then $B$ is a reduction algebra, and the $B$-invariant subspaces of $H$ are exactly the ranges of the central projections of $\mathcal{A}^{\prime \prime}$.

Proof. Clearly Lat $B=$ Lat $\mathcal{A} \cap$ Lat $\mathcal{A}^{\prime}$. Suppose $V \in$ Lat $\mathcal{A} \cap$ Lat $\mathcal{A}^{\prime}$. Since $H$ has the reduction property as an $\mathcal{A}$-module, there is $W \in$ Lat $\mathcal{A}$ with $V \oplus W=H$. The elements of $\mathcal{A}^{\prime}$ have the matrix form [ $\left[\begin{array}{l}* \\ 0\end{array}\right]$ with respect to this decomposition, where the asterisks denote the possibly non-zero entries. Lemma 4.11 shows that in fact elements of $\mathcal{A}^{\prime}$ are of the form $\left[\begin{array}{l}* \\ 0 \\ 0\end{array}\right]$. Thus the projection $\left[\begin{array}{ll}1 & 0 \\ 0 & 0\end{array}\right]$ lies in $\mathcal{A}^{\prime \prime} \cap \mathcal{A}^{\prime}$, and $V$ is the range of a central projection in $\mathcal{A}^{\prime \prime}$. Conversely, the range of any central projection of $\mathcal{A}^{\prime \prime}$ is clearly in Lat $\mathcal{B}$.

\section{Algebras of compact operators}

The above results allow us to use an extension of Lomonosov's lemma due to Shul'man to show that if $\mathcal{A} \subseteq \mathcal{K}(H)$ is a complete reduction algebra with no proper central projections in $\mathcal{A}^{\prime \prime}$ then Lat $\mathcal{A}$ contains irreducible submodules. This gives us the wedge we need to attack Theorem 4.3.

Shul'man's theorem states that any operator algebra whose radical contains a nonzero compact operator shares a proper invariant subspace with its commutant [13]. To put this theorem to use in our context we need only make the following observation.

LEMMA 5.1. Let $\mathcal{A} \subseteq \mathcal{K}(H)$ be a complete reduction algebra. If Lat $\mathcal{A}$ contains no non-zero irreducible submodules then $\mathcal{A}$ contains only quasinilpotent operators, and is hence a radical algebra.

Proof. Let $N \subseteq$ Lat $\mathcal{A}$ be a maximal chain of submodules of $H$. For $V \in N$ the maximality of $N$ shows that the submodule

$$
V_{-}=\overline{\operatorname{span}}\left\{V^{\prime}: V^{\prime} \in N \text { and } V^{\prime} \subset V\right\}
$$

is either equal to $V$ or a maximal submodule of $V$. Thus if $W$ is a complement to $V_{-}$ in $V$ then $W$ is irreducible. Since there are no non-zero irreducible submodules of $H$ 
it follows that $V_{-}=V$ for all $V \in N$. A theorem of Ringrose [2, Theorem 3.4] now gives that all elements of $\mathcal{A}$ are quasinilpotent.

PROPOSITION 5.2. Let $\mathcal{A} \subseteq \mathcal{K}(H)$ be a complete reduction algebra with no proper central projections in $\mathcal{A}^{\prime \prime}$. Then $H$ contains a non-zero irreducible submodule.

PROOF. If $H$ contains no non-zero irreducible submodules then $\mathcal{A}$ is radical, and so shares a proper invariant subspace with $\mathcal{A}^{\prime}$. From Lemma 4.12 this implies the existence of a proper central projection in $\mathcal{A}^{\prime \prime}$.

The next step is to use Lomonosov's lemma to show that the irreducible representations of $\mathcal{A}$ behave as they ought to.

LEMMA 5.3. Let $\mathcal{A} \subseteq \mathcal{K}(H)$ be an operator algebra with the complete reduction property, and suppose that $V, W \in \mathrm{Lat} \mathcal{A}$. If $V$ is irreducible and $T: V \rightarrow W$ is a non-zero module map, then the range of $T$ is closed and $T$ is an isomorphism onto its range.

PROOF. The (maybe non-closed) restriction algebra $\left.\mathcal{A}\right|_{V}$ is an algebra of compact operators, such that $V$ has no proper closed submodules. Lomonosov's lemma implies that $\left.\mathcal{A}\right|_{V}$ is weakly dense in $\mathcal{B}(V)$, and so $\left(\left.\mathcal{A}\right|_{V}\right)^{\prime}=\mathbb{C} 1_{V}$. Suppose $T: V \rightarrow W$ is a non-zero module map. Replacing $W$ with $\overline{T V}$ we may assume that $T$ has dense range. By Lemma 4.11 there is a non-zero module map $S: W \rightarrow V$. Then $S T: V \rightarrow V$ is a module map, non-zero by the density of $T V$. This means that $0 \neq S T \in\left(\left.\mathcal{A}\right|_{V}\right)^{\prime}=\mathbb{C} 1_{V}$, and so $T$ is bounded below. Thus $T V$ is closed and $T$ is an isomorphism of $V$ onto its range.

LEMMA 5.4. Suppose $\mathcal{A} \subseteq \mathcal{K}(H)$ has the complete reduction property. Let $V \in$ Lat $\mathcal{A}$ be irreducible and $W \in$ Lat $\mathcal{A}$ be arbitrary. There is a non-zero module map $T: W \rightarrow V$ if and only if $W$ contains a submodule isomorphic to $V$.

PROOF. Suppose that $T: W \rightarrow V$ is a non-zero module map. Lemma 4.11 implies that there is a non-zero module map $S: V \rightarrow W$, which is an isomorphism onto its range by Lemma 5.3 .

Conversely, if $V^{\prime} \subseteq W$ is isomorphic to $V$, there is a module projection from $W$ onto $V^{\prime}$. Composing this projection with the isomorphism from $V^{\prime}$ to $V$ yields the required non-zero module map.

LEMMA 5.5. Let $\mathcal{A} \subseteq \mathcal{K}(H)$ be an operator algebra with the complete reduction property. If $V, W \in \mathrm{Lat} \mathcal{A}$ and $V$ is irreducible, then $V+W$ is closed. 
PRoOF. If $V \cap W \neq\{0\}$ then $V \cap W=V$ by irreducibility, and $V+W=W$, which is closed.

If $V \cap W=\{0\}$, find $U \in$ Lat $\mathcal{A}$ such that $U \oplus W=H$, and let $p: H \rightarrow U$ be the projection onto $U$ along $W$. Then $T=\left.p\right|_{V}: V \rightarrow U$ is a non-zero module map, and so, by Lemma 5.3, $T$ is an isomorphism of $V$ onto its range contained in $U$. If $V+W$ is not closed there are $\xi \in V, \eta \in W$ with $\|\xi\|>1$ and $\|\xi+\eta\|<$ $\left\|T^{-1}\right\|^{-1}\|p\|^{-1}$. But then $\|T(\xi)\|=\|p(\xi)\|=\|p(\xi+\eta)\|<\left\|T^{-1}\right\|^{-1}$, whence $1<\|\xi\| \leq\left\|T^{-1}\right\|\|T(\xi)\|<1$. This contradiction implies that $V+W$ is closed.

LEMMA 5.6. Let $\mathcal{A} \subseteq \mathcal{K}(H)$ be a operator algebra with the complete reduction property and suppose $V \in$ Lat $\mathcal{A}$ is an irreducible submodule. Let $W \subseteq H$ be the closed span of a family of submodules of $H$ each isomorphic to $V$. Then $W$ is the direct sum of finitely many submodules isomorphic to $V$.

Proof. Let $V_{1} \subseteq W$ be isomorphic to $V$. We inductively define sequences $\left\{V_{i}\right\}$ and $\left\{W_{i}\right\}$ of submodules as follows:

If $W_{n}=\overline{\operatorname{span}}\left\{V_{i}\right\}_{i \leq n} \neq W$, then by the definition of $W$ there is a submodule $V_{n+1} \subseteq W$, isomorphic to $V$ and not contained in $W_{n}$. Since $V_{n+1}$ is irreducible, we have $V_{n+1} \cap W_{n}=\{0\}$. By Lemma 5.5, the algebraic sum $W_{n}+V_{n+1}$ is closed and so $W_{n}+V_{n+1}=W_{n} \oplus V_{n+1}$.

Using this to define inductively the sequences $\left\{V_{i}\right\}$ and $\left\{W_{i}\right\}$, there are two possibilities: either $W_{n} \varsubsetneqq W$ for all $n$ or $W_{n}=W$ for some $n$. However, the first case cannot occur. To see this, observe that by Lomonosov's lemma the algebra $\left.\mathcal{A}\right|_{V}$ contains a compact operator with non-zero eigenvalues, and hence $\mathcal{A}$ contains an operator with non-zero eigenvalues. Using the holomorphic functional calculus, there is a non-zero finite-rank projection $p \in \mathcal{A}$ with $\left.p\right|_{V} \neq 0$. Since $V_{i}$ is isomorphic to $V$ for all $i$, we have $\left.p\right|_{V_{i}} \neq 0$ and so there can only be finitely many $\left\{V_{i}\right\}$. Now by construction $W=W_{n}=\sum_{i \leq n}^{\oplus} V_{i}$.

LEMma 5.7. Let $\mathcal{A} \subseteq \mathcal{K}(H)$ be a complete reduction algebra and suppose $\mathcal{A}^{\prime \prime}$ contains no central idempotents. Then there exists an irreducible submodule $V \in$ Lat $\mathcal{A}$, and $\mathcal{A}$ is similar to $\mathcal{K}(V) \otimes 1_{n}$ for some $n \in \mathbb{N}$.

Proof. We have already seen that there is an irreducible $V \in$ Lat $\mathcal{A}$. Let $W$ be the closed span of all isomorphic copies of $V$ in Lat $\mathcal{A}$. From Lemma 5.6, $W$ is a finite direct sum of $n$ isomorphic copies of $V$. If $W \neq H$ there is $U \in$ Lat $\mathcal{A}$ with $H=W \oplus U$. By definition $U$ does not contain any isomorphic copies of $V$. In this case the commutant $\mathcal{A}^{\prime}$ can be expressed in matrix form as

$$
\left[\begin{array}{cc}
\left(\left.\mathcal{A}\right|_{W}\right)^{\prime} & 0 \\
0 & \left(\left.\mathcal{A}\right|_{U}\right)^{\prime}
\end{array}\right]
$$


where the zeros are obtained from Lemmas 5.3-5.4. Thus the operator $\left[\begin{array}{ll}1 & 0 \\ 0 & 0\end{array}\right]$ is a central projection of $\mathcal{A}^{\prime \prime}$. This contradiction implies that $W=H$.

This allows us to write $H=\sum_{i \leq n}^{\oplus} V_{i}$, where $V_{i} \cong V$ via module isomorphisms $T_{i}: V_{i} \rightarrow V$. We renorm $H$ by

$$
\left\|\sum^{\oplus} \xi_{i}\right\|_{\text {new }}^{2}=\sum\left\|T_{i}\left(\xi_{i}\right)\right\|^{2} .
$$

This renorming effects a similarity on $H$ which exhibits $\mathcal{A}$ as similar to $\mathcal{K}(V) \otimes 1_{n}$.

Theorem 4.10 and Lemma 5.7 show that $\mathcal{A}$ is similar to a $c_{0}$-direct sum of ideals, each of which is similar to $\mathcal{K}\left(V_{\dot{\lambda}}\right) \otimes 1_{n_{\hat{\lambda}}}$. The only remaining step is to show that the similarities needed for each ideal of $\mathcal{A}$ are uniformly bounded in size, so that they may be strung together to apply to all of $\mathcal{A}$. A modification of Lemma 3.4 provides just what we need.

LEMMA 5.8. Suppose that $\mathcal{A} \subseteq \mathcal{K}(H)$ is a complete reduction algebra with no proper central projections in $\mathcal{A}^{\prime \prime}$, and suppose $H^{(\infty)}$ has projection constant $M$. Then there is a similarity $S$ on $H$ with $\|S\|\left\|S^{-1}\right\| \leq 128 M^{2}$ such that $\mathcal{A}^{S}$ is self-adjoint.

Proof. We know that there is an integer $m$ such that $H$ is the direct sum of $m$ mutually isomorphic irreducible submodules. Let us choose one such submodule $V \subseteq H$, so that $H$ is isomorphic to $V^{(m)}$ as an $\mathcal{A}$-module.

Note that for any integer $n$ the submodules of $V^{(n)}$ are of the form $V \otimes W$ where $W \subseteq \mathbb{C}^{n}$. In particular, all rank $m$ submodules of $V^{(2 m)}$ are isometrically isomorphic to $V^{(m)}$ as $\mathcal{A}$-modules.

Paralleling Lemma 3.4, set

$$
\alpha=\inf \left\{\|S\|\left\|S^{-1}\right\|: S \text { is a module isomorphism between } H \text { and } V^{(m)}\right\},
$$

so there is a contractive module isomorphism $S: H \rightarrow V^{(m)}$ with $\left\|S^{-1}\right\| \leq 2 \alpha$. For $\mu \in \mathbb{R}^{+}$we consider the graph subspace Gr $\mu S \subseteq H \oplus V^{(m)}$. Observe that $H \oplus V^{(m)}$ is embedded isometrically in $H^{(m+1)}$ and so $H \oplus V^{(m)}$ has the reduction property with projection constant $\leq M$.

Now follow the proof of Lemma 3.4, replacing ' $H^{\prime}$ ' with ' $V^{(m)}$ ' and ' $*$-module' with 'module isometrically isomorphic to $V^{(n)}$ for some $n$ ' to obtain the estimate $\alpha \leq 128 M^{2}$.

We may now restate and prove Theorem 4.3.

THEOREM 5.9. Let $\mathcal{A} \subseteq \mathcal{K}(H)$ be an operator algebra. Then $\mathcal{A}$ has the complete reduction property if and only if $\mathcal{A}$ is similar to a $C^{*}$-algebra. 
Proof. Suppose that $\mathcal{A} \subseteq \mathcal{K}(H)$ has the complete reduction property. If $\mathcal{A}$ does not act nondegenerately, there is a submodule $V \in$ Lat $\mathcal{A}$ with $\overline{\mathcal{A H}} \oplus V=H$ and $\mathcal{A} V=\{0\}$. Applying a similarity we may assume that $\overline{\mathcal{A} H} \perp V$. Then $\mathcal{A}$ will be self-adjoint if and only if $\left.\mathcal{A}\right|_{\overline{\mathcal{A H}}}$ is self-adjoint, so we may assume that $\mathcal{A}$ acts nondegenerately.

By Theorem 4.10, we may assume that there is an orthogonal family $H_{\lambda}$ of submodules of $H$ with $H=\sum^{\oplus} H_{\lambda}$, and $\mathcal{A}=\sum^{c_{0}} \mathcal{A}_{\lambda}$, where $\mathcal{A}_{\lambda} \subseteq \mathcal{K}\left(H_{\lambda}\right)$ are complete reduction algebras such that the algebras $\mathcal{A}_{\lambda}^{\prime \prime}$ have no proper central projections. By Lemma 5.7, for each $\lambda$ there is a submodule $V_{\lambda} \subseteq H_{\lambda}$ and integer $n_{\lambda}$ such that $\left.\mathcal{A}_{\lambda}\right|_{V_{\lambda}}=\mathcal{K}\left(V_{\lambda}\right)$ and $\mathcal{A}_{\lambda} \cong \mathcal{K}\left(V_{\lambda}\right) \otimes 1_{n_{\lambda}}$. Moreover, by Lemma 5.8 the similarities $S_{\lambda} \in \mathcal{B}\left(H_{\lambda}\right)$ needed to make $\mathcal{A}_{\lambda}$ self-adjoint can be chosen uniformly bounded. The direct sum similarity $S=\sum^{\oplus} S_{\lambda}$ will orthogonalise $\mathcal{A}$.

Since all self-adjoint operator algebras have the complete reduction property the converse is immediate.

COROLlaRY 5.10. Let $\mathcal{A} \subseteq \mathcal{K}(H)$. Then $\mathcal{A}$ is a total reduction algebra if and only if $\mathcal{A}$ is similar to a $C^{*}$-algebra.

Proof. Suppose that $\mathcal{A}$ is a total reduction algebra. Certainly $\mathcal{A}$ is a complete reduction algebra so by Theorem 5.9 is similar to a $C^{*}$-algebra. By the structure theorem discussed above, $\mathcal{A}$ is thus amenable, and Proposition 2.2 completes the argument.

Note that the argument here shows that an operator algebra $\mathcal{A} \subseteq \mathcal{K}(H)$ is amenable if and only if it is similar to a $C^{*}$-algebra. In particular, it follows that an operator in $\mathcal{K}(H)$ generates an amenable algebra if and only if it is similar to a normal operator, a result due to Willis [14]. More generally, it has recently been shown in [4] that a general $T \in \mathcal{B}(H)$ generates an amenable algebra $\mathcal{A}$ if and only if $T$ is similar to a normal operator whose spectrum has connected complement and empty interior (in which case $\mathcal{A}$ is similar to a $C^{*}$-algebra).

\section{References}

[1] W. Arveson, An invitation to $C^{*}$-algebra (Springer, New York, 1976).

[2] K. R. Davidson, Nest algebras (Longman Scientific and Technical, Essex, 1988).

[3] M. M. Day, Normed linear spaces, 3rd edition (Springer, Berlin, 1973).

[4] D. R. Farenick, B. E. Forrest and L. Marcoux, 'Amenable operators on Hilbert spaces', J. Reine Angew: Math. 582 (2005), 201-228.

[5] U. Haagerup, 'Solution of the similarity problem for cyclic representations of $C^{*}$-algebras', Ann. of Math. (2) 118 (1983), 215-240. 
[6] B. E. Johnson, Cohomology in Banach algebras, Mem. Amer. Math. Soc. 127 (Amer. Math. Soc., Providence, RI, 1972).

[7] R. V. Kadison, 'On the orthogonalization of operator representations', Amer. J. Math. 77 (1955), $600-620$.

[8] A. T.-M. Lau, R. J. Loy and G. A. Willis, 'Amenability of Banach and $C^{*}$-algebras on locally compact groups', Studia Math. 119 (1996), 161-178.

[9] V. Paulsen, Completely bounded maps and dilations (Longman Scientific and Technical, Essex, 1986).

[10] G. Pisier, Similarity problems and completely bounded maps, Lecture Notes in Math. 1 (Springer, 1996)

[11] H. Radjavi and P. Rosenthal, Invariant subspaces (Springer, Berlin, 1973).

[12] S. Rosenoer, 'Completely reducible algebras containing compact operators', J. Operator Theory 29 (1993), 269-285.

[13] V. S. Shul'man, 'Invariant subspaces of Volterra operators', Funct. Anal. Appl. 18(1984), 160-161.

[14] G. A. Willis, 'When the algebra generated by an operator is amenable', J. Operator Theory 34 (1995), 239-249.

Mathematical Sciences Institute

Australian National University

Canberra, ACT 0200

Australia

e-mail: giffordj@maths.anu.edu.au 